\title{
High Regression Rate Hybrid Rocket Fuel Grains with Helical Port Structures
}

\author{
Stephen A. Whitmore,,- Sean D. Walker, $₫$ Daniel P. Merkley, $₫$ and Mansour Sobbi \\ Utah State University, Logan, Utah 84322-4130
}

DOI: $\underline{10.2514 / 1 . B 35615}$

\begin{abstract}
Results from a development campaign, where modern additive manufacturing methods are used to fabricate hybrid rocket fuel grains with embedded helical ports, are presented. The fuel grains were constructed from acrlyonitrile butadiene styrene using commercially available three-dimensional printer feedstock material. Gaseous oxygen is used as the oxidizer for this test campaign. When compared to cylindrical fuel ports, significant increases in fuel regression rates were observed, and these increases in regression rate diminished with time as the helical fuel port burns to become progressively more cylindrical. Comparisons to the helical pipe flow skin friction correlation developed by Mishra and Gupta indicate that increased skin friction only partially accounts for the increased regression rate. The Mishra-Gupta correlation underpredicts the regression rate amplification early in the fuel burn and overpredicts that value late in the burn. The authors conclude that radial wall-blowing due to fuel pyrolysis is suppressed by centrifugal forces introduced by the helical flow and compresses the boundary-layer flame zone closer to the fuel port wall. Thus, convective heat transfer is significantly enhanced, and that effect also contributes significantly to the observed regression rate increase. This effect diminishes with time and is not modeled by the Mishra-Gupta correlation.
\end{abstract}

5
$A_{\text {burn }}$
$A_{c}$
$A^{*}$
$C_{f_{x}}$
$D$
$D_{\text {eq }}$
$D_{\text {final }}$
$D_{0}$
$d$
$G_{\text {ox }}$
$\bar{G}_{\text {total }}$
$h_{v}$
$I_{\text {sp }}$
$L_{\text {port }}$
$M_{W}$
$\dot{m}_{\text {fuel }}$
$\dot{m}_{\text {propellant }}$
$\dot{m}_{\text {ox }}$
$N$
$O / F$
$P$
$P_{r}$
$P_{0}$
$R_{c}$
$R e$

\section{Nomenclature}

$=$ fuel grain surface burn area, $\mathrm{cm}^{2}$

$=$ fuel chamber cross-sectional area, $\mathrm{cm}^{2}$

$=$ nozzle throat area, sonic choke area, $\mathrm{cm}^{2}$

$=$ local skin friction coefficient

$=$ instantaneous port diameter, $\mathrm{cm}$

= equivalent diameter based on cross-section area, $\mathrm{cm}$

final port diameter, $\mathrm{cm}$

$=$ initial fuel port diameter, $\mathrm{cm}$

$=$ helix loop diameter, $\mathrm{cm}$

$=$ oxizider mass flux, $\mathrm{kg} / \mathrm{s} \cdot \mathrm{m}^{2}$

$=$ longitudinal mean of total fuel port mass flux, $\mathrm{kg} / \mathrm{s} \cdot \mathrm{m}^{2}$

$=$ mass-specific enthalpy of gasification of fuel material, $\mathrm{J} / \mathrm{kg}$

$=$ specific impulse, $\mathrm{s}$

$=$ fuel port length, $\mathrm{m}$

$=$ molecular weight of combustion products, $\mathrm{kg} / \mathrm{kg}$. mol

$=$ solid fuel grain mass flow rate, $\mathrm{kg} / \mathrm{s}$

$=$ propellant mass flow rate, $\mathrm{kg} / \mathrm{s}$

$=$ oxidizer mass flow rate, $\mathrm{kg} / \mathrm{s}$

$=$ number of helix rotations

$=$ oxidizer to fuel mass flow ratio

$=$ helix pitch length, $\mathrm{cm}$

$=$ Prandtl number

$=$ combustion chamber pressure, $\mathrm{kPa}$

$=$ helix radius of curvature, $\mathrm{cm}$

$=$ longitudinal Reynolds number
Presented as Paper 2014-3751 at the 50th AIAA/ASME/SAE/ASEE Joint Propulsion Conference, Cleveland, OH, 28-30 September 2014; received 28 September 2014; revision received 16 February 2015; accepted for publication 5 May 2015; published online XX epubMonth XXXX. Copyright (C) 2015 by Utah State University. Published by the American Institute of Aeronautics and Astronautics, Inc., with permission. Copies of this paper may be made for personal or internal use, on condition that the copier pay the $\$ 10.00$ per-copy fee to the Copyright Clearance Center, Inc., 222 Rosewood Drive, Danvers, MA 01923; include the code 1533-3876/YY and $\$ 10.00$ in correspondence with the CCC.

*Associate Professor, Mechanical and Aerospace Engineering Department, 4130 Old Main Hill, UMC 4130. Associate Fellow AIAA.

${ }^{\dagger}$ Graduate Research Associate, Mechanical and Aerospace Engineering Department, 4130 Old Main Hill, UMC 4130. Student Member AIAA.

${ }^{\ddagger}$ Graduate Research Assistant, Mechanical and Aerospace Engineering Department, 4130 Old Main Hill, UMC 4130. Student Member AIAA.

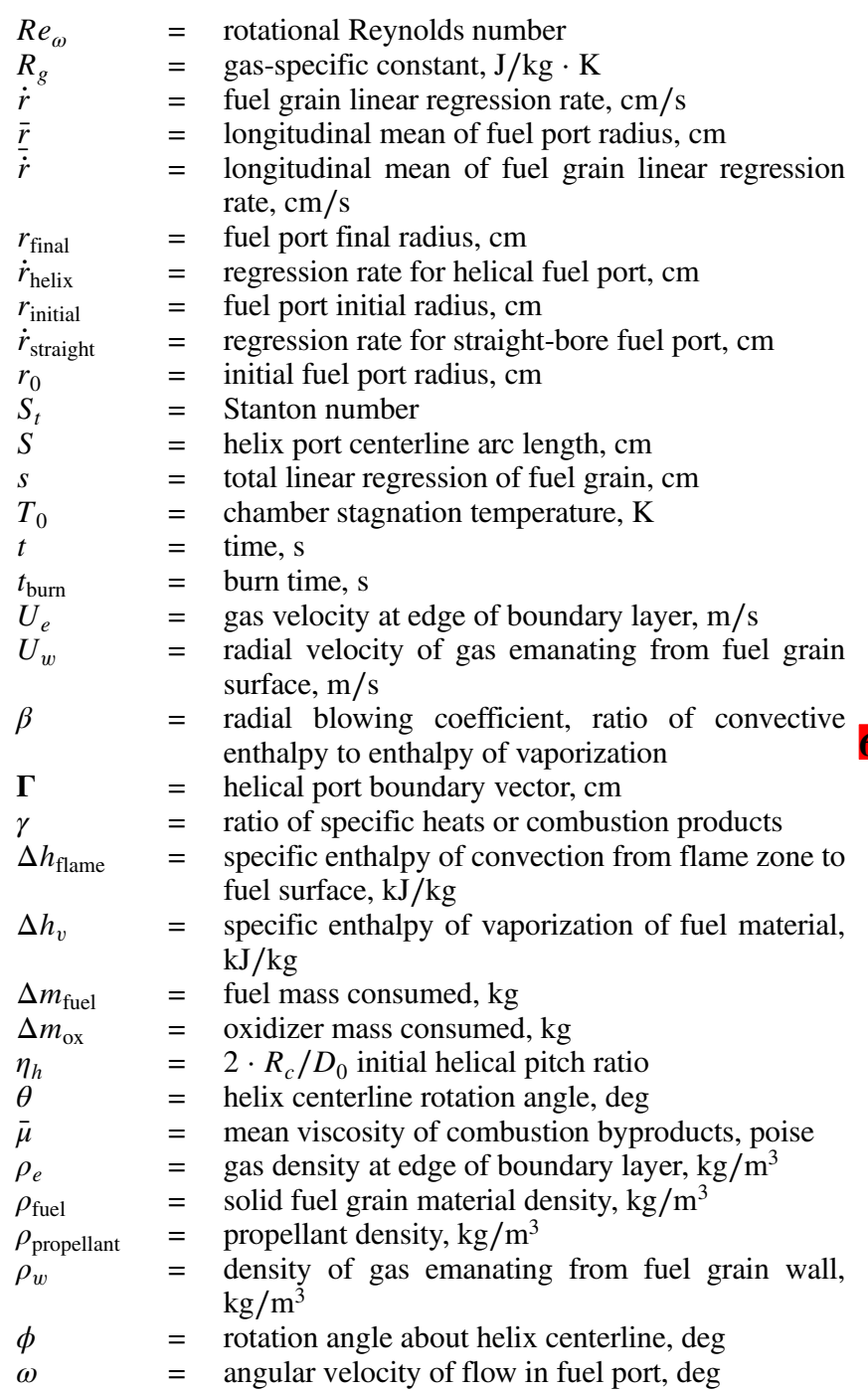

\section{Introduction}

H YBRID rocket motors, in spite of their well-known safety and handling advantages [1], have not seen widespread commercial 
use due to internal motor ballistics [2] that produce fuel regression rates typically $25-30 \%$ lower than solid fuel motors in the same thrust and impulse class. These lowered fuel regression rates tend to produce unacceptably high oxidizer-to-fuel $(O / F)$ ratios that lead to potential combustion instability, erosive burning, nozzle erosion, and reduced motor duty cycles. To achieve $O / F$ ratios with acceptable combustion characteristics, traditional cylindrical fuel ports are fabricated to very long length-to-diameter ratios. These high-aspectratio motors result in poor volumetric efficiency and present the potential for lateral structural loading issues during high thrust burns.

By increasing the oxidizer mass flux, the fuel regression rate can theoretically be increased; however, the resulting combustion instabilities at high flux rates limit the effectiveness of this option [3]. To achieve enhanced fuel mass flows for lower oxidizer mass flux levels, hybrid fuel grain designers have resorted to increasing the fuel grain surface burn area by casting multiple fuel ports with a large precombustion chamber or multiple injectors or by increasing the fuel port length. The original AMROC 15-port grain design is a classic realization of this high-surface-area technique [4]. There are several disadvantages to the multiple-port approach [5]. First, the overall fuel regression rate is reduced as the number of ports increases and the motor diameter size grows accordingly. Second, there is a significant potential for uneven port burning. Uneven burning presents a significant potential for compromised fuel grain integrity, especially toward the end of the burn. Also, multiport fuel grain designs typically produce unburned mass fraction of greater than $10 \%$. Third, multiple-port designs present an increased risk of feed-coupling instabilities related to dynamic flow interactions between the injector (s), the multiple fuel ports, and the precombustion chamber [3]. Finally, complex casting multiport geometries using conventional propellants requires the development of extensive tooling and presents an unavoidable difficulty with removing the tooling once the grain material is cured. There is often a requirement for an embedded structure to support the fuel port as it regresses. The presence of this supporting web adds complexity to the fuel port design and has the potential to allow voids within the fuel grain structure. Such voids can contribute to the potential for fuel grain fractures [6]

Other techniques for increasing fuel regression rates, generally based on increasing the heat transfer from the combustion zone to the fuel grain surface, have been tested [7]. Unfortunately, most of these methods suffer significant operational shortcomings. These techniques include adding oxidizing agents to the fuel material [8], adding metal particles to the fuel grain [9], and the use of swirl injection to increase the local oxidizer surface mass flux [10]. All of these techniques have demonstrated some ability to enhance regression rates but have also introduced multiple disadvantages.

Introducing oxidizing materials into the fuel grain reverts to a quasi-solid propellant design and introduces the potential for pressure coupling during the motor burn [11]. Pressure coupling causes a significant increase in the fuel regression rate as the motor chamber pressure increases. Hybrid rocket combustion frequently displays a sudden amplification of combustion pressures leading into lowfrequency instability that typically occurs in the $10-20 \mathrm{~Hz}$ range $[12,13]$. Thus, introducing pressure coupling can result is a significantly increased explosion risk.

Introducing micron-sized metal particles can also introduce pressure coupling, and the resulting increase in the effective exhaust product molecular weights results in only marginal end-to-end motor performance improvements, especially when higher-performance oxidizers are used [14]. Introducing nanosized metal particles increases motor production costs, and uniform fuel grain material properties are more difficult to achieve.

Finally, swirl injection has been demonstrated to be effective in increasing the fuel regression rate $[15,16]$. Swirl injectors are able to reduce both thickness and growth of the boundary layer, thus enhancing heat transfer. The heat transfer variation is reduced, and regression rate is more uniform. Some effects of swirling must be addressed, including induced torque and effects of nonaxial flow in the nozzle (effective throat area and divergence losses). No swirl or vortex injection hybrid motor has ever been flight-tested. The effects of motor acceleration upon the swirl effectiveness has yet to be assessed.

Karabeyoglu et al. [17] have investigated a class of hybrid fuel materials based on paraffin wax formulations. These paraffin-based fuels melt before vaporizing, and a properly formulated wax mix produces a melt layer with a low viscosity and high surface tension. When the oxidizer flows at high speed over the upper side of the melting fuel surface, the liquid layer becomes unstable, and minute surface waves are formed. The resulting fluid boundary layer is hydrodynamically unstable and allows fuel droplets to be entrained into the core flow. The entrained fluid droplets significantly increase the mass flow generated by the ablating fuel but do not increase the "blowing effect" that suppresses regression rate due to the ablating radial mass flow. For stable oxidizer flux levels, droplet entrainment mass flow is significantly greater than mass flow resulting from direct gasification. Paraffin-based fuels have been developed that burn at surface regression rates three to four times that of conventional hybrid fuels [18]. The high regression rate hybrid removes the need for a complex multiport grain, and most applications up to large boosters can be designed with a single-port configuration. Karabeyoglu et al. have ground-tested paraffin fuel hybrid rocket motors large as $60 \mathrm{~cm}$ in diameter [19].

However, because of the fuel drop entrainment, significant unburned materials are ejected from the nozzle, and combustion efficiencies for paraffin-based fuels are inherently lower. More significantly, the properties that allow the fuel droplet entrainment in paraffin-based fuels introduce mechanical and structural problems that reduce the fuel grain integrity as the propellant burns. Solid phase paraffin is rather brittle and is easily cracked when subjected to launch vibration loads. As the paraffin melts, the material softens and tends to flow and "sluff" under axial launch loads. Thus, paraffinbased fuels require either special additives or a support lattice to keep the grain structure intact under launch loads.

Several strengthening materials have been tested in hybrid motors [20]. Polyurethane foam (PUF) strengthening structure shows promising results but leads to heterogeneous fuel formulations that are difficult to manufacture with any degree of consistency. To avoid this problem and to ensure paraffin-based formulations with sufficient elasticity to survive launch vibration levels, a miscible thermoplastic elastomer styrene-ethylene-butylene-styrene (SEBS) was tested as a strengthening alternative to PUF. Mixing SEBS into the paraffin fuel produces a homogenous fuel grain and offers significantly lower manufacturing costs. During the combustion of the homogeneous material, the material melts; when using heterogeneous materials, only the paraffin melts. Unfortunately, both the SEBS fuel additive and PUF structural support materials reduced the burn effectiveness and performance of the hybrid motor.

The vast majority of conventional hybrid rocket motor designs use the isocyanate-cured thermosetting polymers hydroxyl-terminated polybutadiene (HTPB), polybutadiene acrylonitrile, and glycidyl azide polymer as the fuel grain material. These fuels are legacy materials derived from solid propellant and explosive ordnance manufacture. The U.S. Department of Defense considers these materials to be environmentally unsustainable for large-scale propellant production and is actively seeking replacement alternatives [21]. The isocyanate curatives present a wide variety of environmental safety and occupational health risks including irritation of the skin, mucous membranes, eyes, and respiratory tract; contact and allergic dermatitis; hypersensitivity pneumonitis; and respiratory sensitization. Some studies have demonstrated that isocyanates also exhibit carcinogenic and detrimental reproductive effects.

In typical thermosetting polymer motor construction, binder materials are mixed from liquid base components, degassed under vacuum, and then cast and cured in a fuel grain mold. Because the base materials must be cast using tooling and mandrels to set the port geometries, there are limitations to the types of port geometries that can be developed. These cast-and-cure methods for producing hybrid fuel grain are necessarily labor intensive, and high production rates cannot be achieved without a significant manufacturing infrastructure. Once cured, thermosetting polymers cannot be remanufactured into another component or recycled. 
Whitmore et al. [22] at Utah State University have recently investigated the use of acrlyonitrile butadiene styrene (ABS) thermoplastic as a hybrid rocket fuel material. A key outcome of this research was the demonstrated thermodynamic equivalence of ABS to HTPB when burned with nitrous oxide $\left(\mathrm{N}_{2} \mathrm{O}\right)$. In a series of comparison tests, it was discovered that the combustion flame temperature for $\mathrm{N}_{2} \mathrm{O} / \mathrm{ABS}$ is slightly cooler than $\mathrm{N}_{2} \mathrm{O} / \mathrm{HTPB}$, but the products of combustion have a lower molecular weight. Thus, ABS achieves specific impulse $I_{\mathrm{sp}}$ and characteristic velocity $c^{*}$ that are nearly identical to HTPB. ABS and HTPB fuel regression rates for cylindrical fuel ports were measured to be statistically identical.

When compared to HTPB, ABS possesses unique material properties that make it very attractive as a hybrid rocket fuel. ABS is a noncrystalline material with an amorphous structure. As such, ABS does not possess a true melting point but exists in a highly softened semifluid state before vaporizing. Depending on the precise material formulation, this intermediate fluid state exists over a temperature range that varies from 120 to $140^{\circ} \mathrm{C}$ [23]. This property makes ABS the material of choice for a modern form of additive manufacturing known as fused deposition modeling (FDM). In FDM, a plastic filament is unwound from a coil and supplies material to an extrusion nozzle. The nozzle is heated to melt the material and can move threedimensionally when controlled by a computer numerically controlled mechanism. Exploiting the FDM fabrication process for ABS offers the potential to revolutionize the manufacture of hybrid rocket fuel grains. FDM can support high production rates and offers the potential of improving hybrid fuel grain quality, consistency, and performance, while reducing development and production costs. Identical grains can be manufactured from a wide range of commercial vendors; thus, FDM processing does not require the maintenance of an application-specific production and assembly line.

Most significantly, using additive manufacturing, ABS fuel grains can be fabricated with an almost infinite range of fuel port shapes. These embedded structures offer the potential for significant fluidmechanical enhancement and modification of burn properties and combustion efficiencies [24]. It is this nearly open-ended manufacturing capability that will be exploited in the research to be detailed in this paper.

\section{Hybrid Fuel Regression Rate Mechanism Overview}

Two fundamentally different processes drive solid and hybrid fuel grain regression rates. Solid propellants are blended using a combination of oxidizer and fuel in a mass proportion that delivers the optimized performance for a given mission requirement. Because the propellant mixture ratio is set by this homogeneous propellant formulation, the $O / F$ remains constant throughout the burn. Solidpropellant combustion occurs in a very thin zone near the fuel surface. The rate of regression is driven by primarily chemical kinetics and is directly coupled to the combustion chamber pressure through the well-known St. Robert's law ([9] Chap. 12).

In contrast to solid rocket motors, where regression rates are strongly coupled to the chamber pressure, hybrid fuel regression rates have little or no dependence on chamber pressure. Thus, hybrid fuel regression rate models based on St. Robert's law are inaccurate. Early studies by Marxman and Gilbert [25] demonstrated that combustion processes for hybrid rockets are mostly driven by the boundary-layer fluid mechanics, where fuel regression rate is a result of turbulent boundary-layer heat transfer. Fuel regression rate is strongly correlated with the oxidizer mass flow through the combustion chamber. Marxman et al. [26] proposed an enthalpy-based regression-rate model, where the convective heat transfer from the combustion flame layer is balanced by the energy of ablation of the fuel grain surface:

$$
\rho_{\text {fuel }} \dot{r} h_{v}=S_{t} \rho_{e} U_{e} \Delta h_{\text {flame }}
$$

In Eq. (1), $\dot{r}$ is the linear regression rate perpendicular to the fuel surface; $\Delta \bar{h}_{\text {flame }}$ is the convective enthalpy transfer per unit mass flow from the flame zone to the fuel surface; $h_{v}$ is the enthalpy of vaporization (latent heat) of the fuel material; $\rho_{\text {fuel }}$ is the density of the solid fuel material; $S_{t}$ is the nondimensional Stanton number; and $\rho_{e} \cdot U_{e} \cong G_{\mathrm{ox}}$ is the oxidizer mass flux at the edge of the boundary layer. In this model, radiation effects and heat conduction within the fuel grain are considered negligible.

The Stanton number can be written in terms of the local skin friction coefficient using the Reynolds-Coburn analogy [27-29]:

$$
S_{t}=\frac{C_{f_{x}}}{2} P_{r}^{-2 / 3}
$$

Substituting Eq. (2) into Eq. (1), the regression rate is written in terms of the local surface skin friction coefficient:

$$
\dot{r}=\left(\frac{G_{\mathrm{ox}}}{2 \cdot P_{r}^{2 / 3} \cdot \rho_{\text {fuel }}}\right) \cdot\left(\frac{\Delta h_{\text {flame }}}{h_{v}}\right) \cdot C_{f_{x}}
$$

In Eqs. (2 , and $\underline{3}$ ), $C_{f_{x}}$ is the local skin friction coefficient, $P_{r}$ is the nondimensional turbulent Prandtl number, and $G_{\mathrm{ox}}$ is the oxidizer mass flow per unit cross-sectional area at the edge of the boundary layer.

\section{A. Effect of Surface Skin Friction on Hybrid Rocket Fuel Regression} Rate

Examining Eq. (3), it becomes clear that there exists a nearly proportional correlation between the local skin friction coefficient and the regression rate of the hybrid fuel grain. Thus, it is reasonable to speculate that any fuel port design feature that increases the local surface skin friction coefficient, such as premanufactured surface indentations or ridges, would also increase the fuel regression rate.

This conclusion is only partially true. In conventional pipe flow, boundary-layer mixing allows the oxidizer flow at the center of the fuel port to directly mix with vaporized material leaving the fuel wall. However, as shown by Fig. 1, for hybrid fuel combustion, the radially emanating flow from the ablating fuel surface expands the boundarylayer thickness and pushes the combustion zone away from the wall. Using Lees's model [30] for the wall blowing coefficient,

$$
\beta=\frac{\rho_{w} \cdot U_{w}}{\rho_{e} \cdot U_{e} \cdot(1 / 2) C_{f_{x}}}
$$

to account for the radially emanating flowfield from the pyrolyzed fuel, Boardman [31] demonstrates that, for hybrid fuel combustion, the radial blowing coefficient is equal to the ratio of the convective heat transfer from the flame zone to the fuel surface and the enthalpy of gasification of the fuel material:

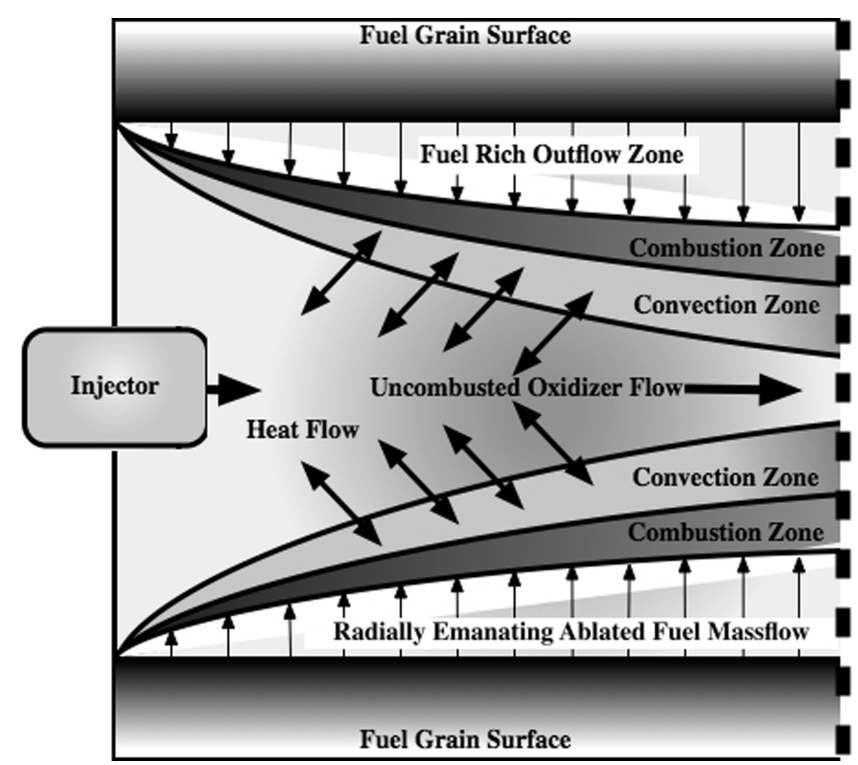

Fig. 1 Enthalpy balance model for hybrid fuel regression rate. 


$$
\beta \approx \frac{\Delta h_{\mathrm{flame}}}{h_{v}}
$$

and Eq. (י) is modified to account for wall blowing according to

$$
\begin{aligned}
\dot{r} & =\left(\frac{G_{\mathrm{ox}}}{2 \cdot P_{r}^{2 / 3} \cdot \rho_{\text {fuel }}}\right) \cdot\left(\frac{\Delta h_{\text {surface }}}{h_{v}}\right) \cdot C_{f_{x}}\left(\frac{1.27}{\beta^{0.77}}\right) \\
& =\left(\frac{0.635 \cdot G_{\text {ox }}}{P_{r}^{2 / 3} \cdot \rho_{\text {fuel }}}\right) \cdot\left(\frac{\Delta h_{\text {surface }}}{h_{v}}\right)^{0.23} \cdot C_{f_{x}}
\end{aligned}
$$

In Eq. (4), $\rho_{w} \cdot U_{w}$ is the mass flux resulting from the radial wall out blowing.

Thus, when flow structures, such as surface roughness, are used to increase the surface skin friction, as the fuel regression rate increases, the blowing coefficient $\beta$ also increases. The result is a flame zone that is pushed away from the fuel surface and a significantly reduced convective heat transfer and rate of enthalpy exchange. When fuel regression rate is initially increased by surface roughness, the additional radially emanating fuel material reduces the effectiveness of the heat transfer mechanism, and diminishing returns rapidly result. It is also very likely that any introduced surface protuberances will lead to erosive burning or be quickly eliminated by the combustion flame.

To significantly increase the fuel regression rate, any proposed port design feature must increase the nominal surface skin friction while also minimizing the effects of radial surface blowing. A helical fuel port structure serves this purpose. Helical fuel ports in a wide variety of cross-sectional areas can be easily manufactured using ABS fuel materials manufactured by FDM techniques.

\section{B. Analytical Model of the Helical Fuel Port}

The helical structure is defined by three parameters: 1 ) the nominal fuel port diameter $D, 2$ ) the helix loop diameter $d$, and 3) the helix pitch length $P$. The pitch length is defined as the distance between the centerlines of two consecutive helical wraps. The pitch length is nominally calculated as the total length of the helix divided by the total rotation angle (radians) along the length of the helix:

$$
P=\frac{L}{N}
$$

The total run length of the helix centerline is

$$
S=2 \pi \cdot N \cdot \sqrt{\left(\frac{\mathrm{d}}{2}\right)^{2}+\left(\frac{P}{2 \pi}\right)^{2}}
$$

In Eqs. (ㄱ, and $\underline{8}), N$ is the number of rotations along the helix length. The radius of curvature of the helical arc is calculated from the loop diameter and pitch length as

$$
R_{c}=\frac{\mathrm{d}}{2} \cdot\left(1+\left(\frac{P}{\pi \cdot \mathrm{d}}\right)^{2}\right)
$$

The initial helical pitch ratio is defined as the two times the radius of curvature divided by the initial port diameter:

$$
\eta_{h}=\frac{2 \cdot R_{c}}{D_{0}}
$$

The polar form of the helical fuel port with a cylindrical cross section may be derived in three dimensions as

$$
\begin{aligned}
& \boldsymbol{\Gamma}(\theta, \phi)=\frac{1}{2} \cdot[\cos (\theta) \cdot[d-D \cdot \cos (\phi)] \\
& \left.+D \cdot \sin (\theta) \cdot \sin (\phi) \cdot \frac{P}{\sqrt{(\pi \cdot \mathrm{d})^{2}+P^{2}}}\right] \cdot \boldsymbol{i} \\
& +\frac{1}{2} \cdot[\sin (\theta)[d-D \cdot \cos (\phi)] \\
& \left.\quad-D \cdot \cos (\theta) \cdot \sin (\phi) \cdot \frac{P}{\sqrt{(\pi \cdot \mathrm{d})+P^{2}}}\right] \cdot \boldsymbol{j} \\
& \quad+\left[\left(\frac{P}{2 \cdot \pi}\right) \cdot \theta+R \cdot \sin (\phi) \cdot \frac{\pi \cdot \mathrm{d}}{\sqrt{(\pi \cdot \mathrm{d})^{2}+P^{2}}}\right] \cdot \boldsymbol{k}
\end{aligned}
$$

In Eq. $(11), \Gamma(\theta, \phi)$ is the vector describing the helical port boundary; $\bar{\theta}$ is the helix centerline rotation angle; $\phi$ is the rotation angle about the helix centerline; and $\{\boldsymbol{i}, \boldsymbol{j}, \boldsymbol{k}\}$ are the unit vectors in the longitudinal, lateral, and normal vectors. The coordinate system is aligned with $\boldsymbol{i}$ pointing along the centerline of the helix. The angles $\theta$ and $\phi$ are rotated from $\theta=\{0,2 \pi \cdot N\}$ and $\phi=\{0,2 \pi\}$ to form the inner boundary of the fuel port. The instantaneous port diameter $D$ is measured perpendicular to the helix centerline and is related to the initial port diameter, the fuel regression rate, and the fuel burn time by

$$
D=D_{0}+2 \cdot \dot{r} \cdot t_{\text {burn }}
$$

Based on the model of Eq. (12), it is interesting to note that, for a linearly regressing fuel grain, the fuel port becomes less and less helical with time. Figure 2 illustrates this result, where a short-pitch helical port cross section is shown for three different mean total regression values, representing the fuel grain as the port burns and opens up. The plotted port cross sections are for regression ratios $s / R_{c}=\{0,1.25,2.5\}$. The symbol $s$ is the linear regression of the fuel port calculated as the difference between the instantaneous and initial mean fuel port radius. Clearly, the final port cross section is no longer helical, and the effective radius of curvature becomes very large. With this large radius of curvature, the helical wall boundary can no longer support a well-defined rotational flow. This property means that the helical fuel port regression rate will slow significantly after a period of burn where the fuel port has become effectively cylindrical. This property also reduces the potential for unburned fuel slivers as the port approaches the outer wall boundary. This effect will be illustrated later in this paper by showing images of the port cross sections on burned fuel grains.

\section{Regression Rate Amplification Model Based on Mishra-Gupta Skin Friction Correlation}

It is well known that helical pipe flows have the effect of significantly increasing the local skin friction coefficient. Helical

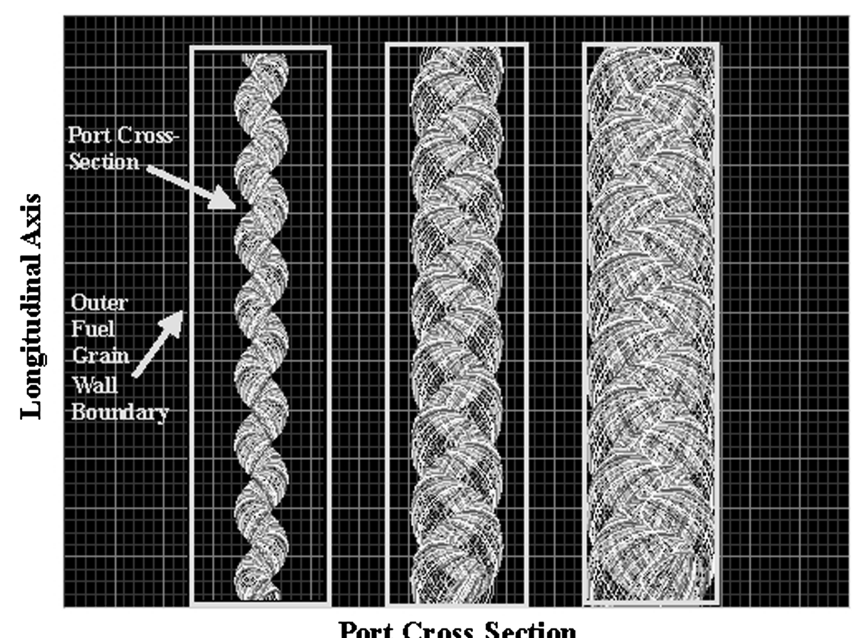

Fig. 2 Fuel port cross sections for three different mean regression values. . 
flows also introduce a centrifugal component into the flowfield. In hybrid rocket applications, this centrifugal component will have the effect of thinning the wall boundary layer, bringing the flame zone closer to the wall surface and increasing the flame diffusion efficiency.

There exist several well-known correlations of increasing fidelity comparing skin friction in helical pipe flows to straight pipe flows. An early correlation was developed by White [32] for smooth coiled tubes and was valid over a Reynolds number range from 15,000 to 500,000 (based on pipe diameter). Ito [33] theoretically derived a similar expression for considerably lower Reynolds numbers than were investigated by White [32]. Gnielinski [34] extended the range of validity of Ito's model for nearly the entire turbulent flow regime.

The models presented by White [32], Ito [33], and Gnielinski [34] were derived for helical coils where the pitch length was significantly smaller than the coil diameter. Thus, these correlations depend only upon the ratio of the pipe inner diameter to the coil diameter and not up on the pitch length of the helix. In a less well-known study, Mishra and Gupta [35] extended the model to account for longer pitch lengths by replacing the coil radius by the radius of curvature of the helix, $R_{c}$. Mishra and Gupta investigated a wide range of coil geometries for both laminar and turbulent flow conditions and derived a "universal" flow correlation of the form

$$
C_{f_{\text {helix }}}=C_{f_{\text {straight }}}+0.0075 \cdot \sqrt{\frac{D}{2 \cdot R_{c}}}
$$

where $C_{f_{\text {helix }}}$ is the skin friction coefficient for the helical coil, and $C_{f_{\text {straight }}}$ is the skin friction coefficient for the straight-bore tube. For fully developed flow in smooth pipes with short pitch lengths, the Mishra and Gupta model produces identical results to Ito's model.

Considering the form of Eq. (13), it is accurate to conclude that the growth in regression rate of the helical fuel port is proportional to growth in the end-to-end skin friction coefficient. Thus, using the Mishra and Gupta model for the helical/straight-bore skin friction ratio and the Schoenherr-Schlicting model for the straight-bore skin friction coefficient, the regression rate "amplification factor", defined as the ratio of the helical and straight-bore fuel port skin friction coefficients, can be approximated as

$$
\begin{aligned}
\dot{r}_{\text {helix }} & =\left(\frac{G_{\mathrm{ox}}}{2 \cdot P_{r}^{2 / 3} \cdot \rho_{\text {fuel }}}\right) \cdot\left(\frac{\Delta h_{\text {surface }}}{h_{v}}\right) \\
& \cdot\left(C_{f_{\text {straight }}}+0.0075 \cdot \sqrt{\frac{D}{2 \cdot R_{c}}}\right) \cdot\left(\frac{1.27}{\beta^{0.77}}\right)
\end{aligned}
$$

In the model of Eq. (14), $D$ and $R_{c}$ are the instantaneous mean port diameter and radius of curvature. Because the model of Eq. (14) is developed from correlations for conventional pipe flow, this model does not account for any suppression of radial wall blowing effects.

An order of magnitude analysis of Eq. (14) shows that, for typical hybrid combustion mass flux rates and Reynolds number, the magnitude of the helical correction parameter

$$
0.0075 \cdot \sqrt{\frac{D}{2 \cdot R_{c}}}
$$

is typically equal to or greater than the magnitude of the local skin friction coefficient based on the port cross section $C_{f_{\text {straight }}}$. Thus, there exists a significant potential for the helical port to significantly increase the overall fuel regression rate.

\section{Previous Helical Fuel Port Studies}

Bath [36] at Utah State University investigated the use of threedimensional FDM printing to build ABS grains with complex port geometries and observed the effects of these geometries on the fuel burn properties. Fuller et al. [24] at Aerospace Corporation have also achieved success with burning helical structures that were fabricated using additive manufacturing techniques. Arnold et al. at Pennsylvania State University have also successfully tested additively manufactured hybrid rocket fuel grains with embedded swirl patterns [37]. The additive manufacturing process used by $[24,37]$ was stereolithography and not FDM. Although quantitative regression rate amplification factors were not published by these early studies, all results demonstrated the qualitative effectiveness of embedded helical port structures in increasing regression rate and thus support the conclusions derived from the order of magnitude analysis of the simple correlation model of Eq. (14).

\section{Experimental Verification of Helix Amplification Factors}

This section reports on a series of experiments conducted to quantitatively investigate the helical regression-rate increase predicted by the order of magnitude analysis of the Mishra-Gupta model as presented in the previous section. This testing campaign used an existing lab-scale motor to perform multiple test firings with both straight-bore and helical port geometries. Gaseous oxygen (GOX) was selected as the initial test oxidizer to allow a pressure-reducing regulator setting that choked the inlet oxidizer flow, thus decoupling the oxidizer mass flow from the combustion chamber pressure. Using GOX also presents the advantage of working with a single-phase oxidizer requiring no thermal conditioning, making the test results much less susceptible to external environmental effects.

\section{A. Test Apparatus for Gaseous Oxygen-Acrlyonitrile Butadiene Styrene Motor Tests}

An existing mobile test stand, the Kart for Reactive Monopropellant Testing [38] was used to perform the test and evaluation campaign. Acquired measurement channels included thrust, chamber pressure, upstream and downstream coolant temperatures, ignitor case temperature, Venturi pressures (inlet and throat) for oxidizer mass flow, and Venturi temperature (necessary for determining GOX density in the Venturi). These fuel regression tests were performed using a well-characterized motor that had been previously used for paraffin fuel regression tests. The motor was adapted from a commercially available $75 \mathrm{~mm}$ Cesaroni Pro75 [39] motor case with a

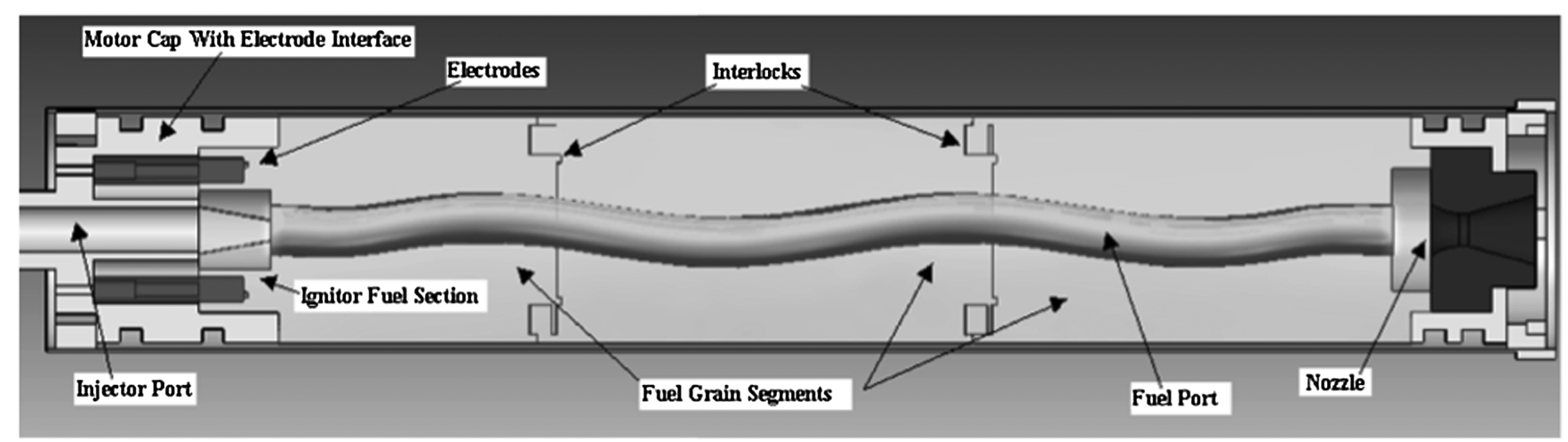

Fig. 3 Schematic of lab-scale hybrid motor with snap-together helical fuel grains. 
Table 1 Fuel grain geometry parameters for GOX-ABS tests

\begin{tabular}{lccccccc}
\hline \hline $\begin{array}{l}\text { Grain } \\
\text { number }\end{array}$ & $\begin{array}{c}\text { Initial } \\
\text { length, cm }\end{array}$ & $\begin{array}{c}\text { Initial port } \\
\text { radius, cm }\end{array}$ & $\begin{array}{c}\text { Initial helix } \\
\text { radius, cm }\end{array}$ & $\begin{array}{c}\text { Initial helix pitch } \\
\text { length, cm }\end{array}$ & $\begin{array}{c}\eta_{h}=\frac{2 \cdot R_{c}}{\Omega_{0}} \\
\text { of burns }\end{array}$ & $\begin{array}{c}\text { Number } \\
\text { grain mass, g }\end{array}$ \\
\hline 1 & 35.98 & 1.013 & -- & -- & -- & 3 & 1147.0 \\
2 & 35.98 & 0.762 & 0.381 & 15.24 & 10.382 & 5 & 1337.0 \\
3 & 35.98 & 0.762 & 0.762 & 15.24 & 5.567 & 2 & 1326.0 \\
4 & 22.86 & 0.762 & 0.5715 & 2.70 & 0.587 & 6 & 838.0 \\
\hline \hline
\end{tabular}

custom-built injector cap, nozzle, and retaining ring. The system featured a novel nonpyrotechnic arc-ignition system developed at Utah State as a part of an earlier additive manufacturing campaign [40]. This ignition system allows multiple consecutive static firings to be performed with no hardware changeover.

The motor design takes advantage of FDM processing to build the ignitor and fuel grain sections with "snap-together" interlocks that allow multiple grain segments to be manufactured

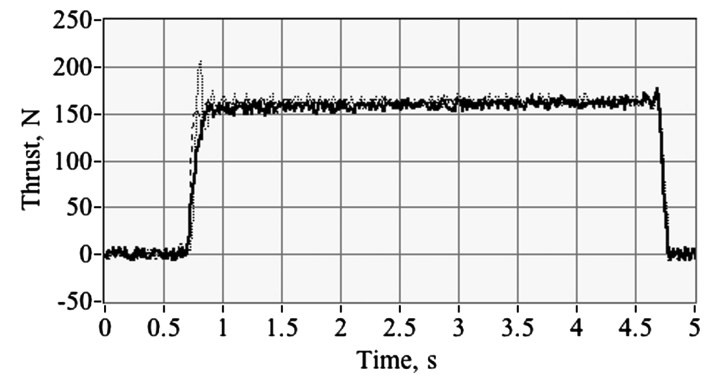

a) Thrust, cylindrical port (grain 1)

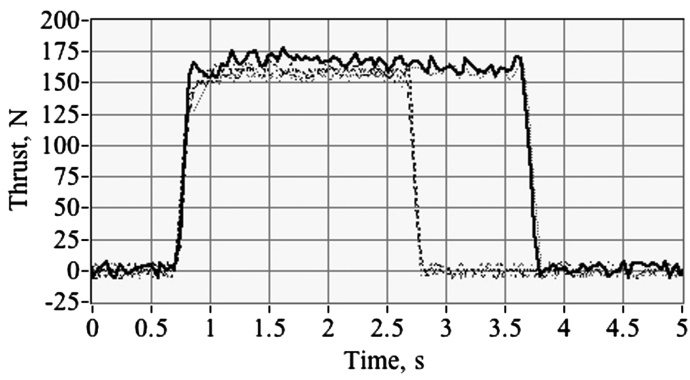

b) Thrust, helix ratio $=\mathbf{1 0 . 3 8}($ grain 2$)$

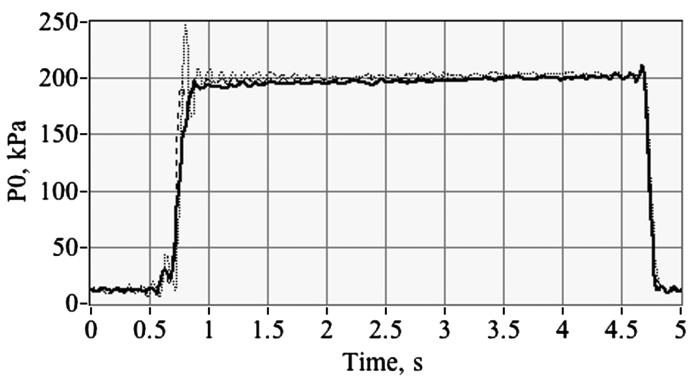

e) Chamber pressure cylindrical port (grain 1)

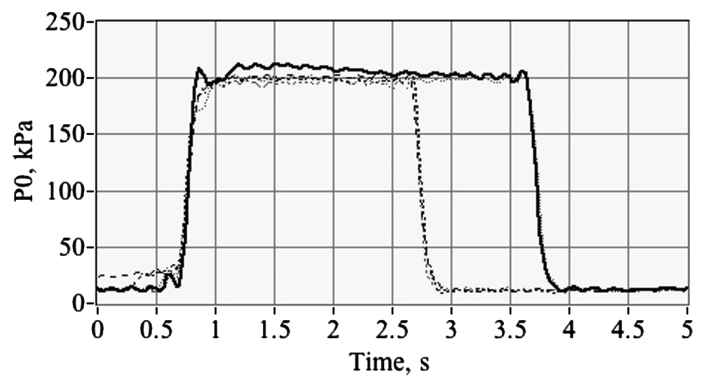

f) Chamber pressure helix ratio $=\mathbf{1 0 . 3 8}$ (grain 2) and then assembled for combustion. The multiple grain segments were simultaneously manufactured on a Stratasys Dimension 1200es three-dimensional (3-D) FDM printer [41] using their ABSplusP430 feedstock material $\left(0.971 \mathrm{~g} / \mathrm{cm}^{3}\right.$ density) [42]. Joints were bonded using commercial grade ABS pipe joint cement. Figure $\underline{3}$ shows the integrated motor system featuring the injector cap, snaptogether fuel grain with the helical port structure, motor case, and nozzle.

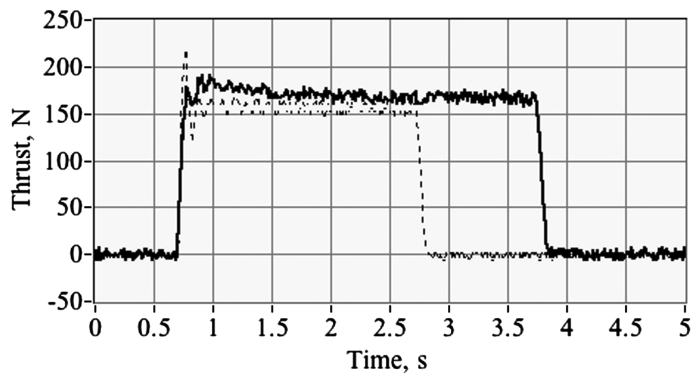

c) Thrust, helix ratio $=\mathbf{5 . 5 7}($ grain 3 )

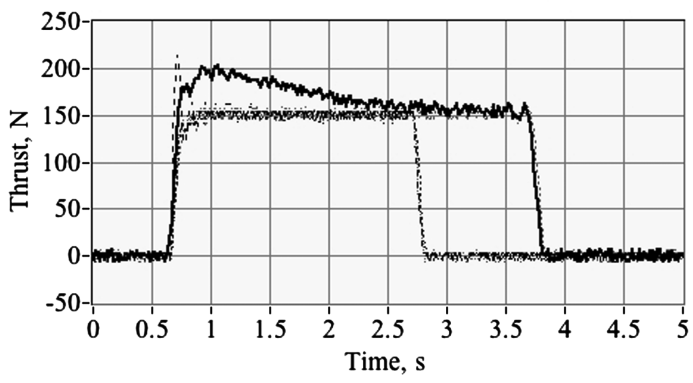

d) Thrust, helix ratio $=0.59$ (grain 4)

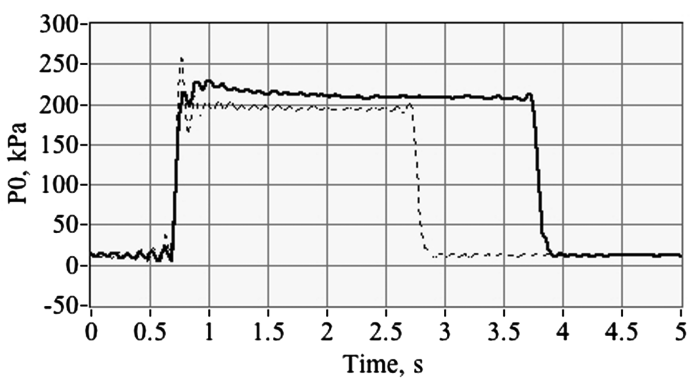

g) Chamber pressure helix ratio $=5.57$ (grain 3)

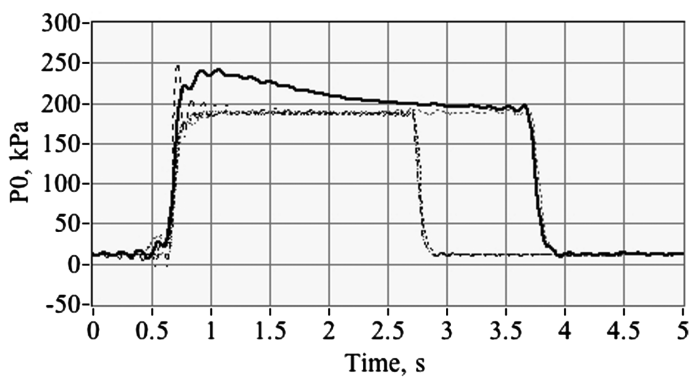

h) Chamber pressure helix ratio $=0.59($ grain 4$)$

Fig. 4 GOX-ABS regression tests, thrust, and chamber pressure time histories. 


\section{B. Test Summary}

Four-fuel grain geometries, a straight-bore cylindrical grain, and three helical ports with varying helix geometries were tested. Table 1 summarizes the parameters of these fuel grains. Real-time thruststand motor mass measurements were considered too noisy to be useful, and so for this testing campaign, each grain was burned multiple times to allow intermediate mass measurements between burns. The straight-bore port tests, where the regression rate was expected to be lowest and the risk of wall burn-through less, were performed with $4 \mathrm{~s}$ burn durations. The helical port tests, where the risk of wall burn-through was significantly greater, were performed with 2 and $3 \mathrm{~s}$ burn durations. Grains sections were inspected between burns to ensure that burn-through had not occurred and that sufficient fuel remained to allow additional burns. Generally, the shorterduration burns were performed last as a precaution against fuel wall burn-through.

In all, 16 static firings were performed as a part of this testing campaign. Figure $\underline{4}$ compares the thrust and chamber pressure pro-

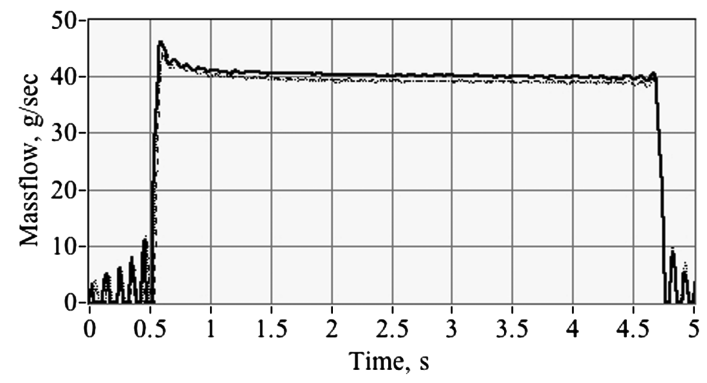

a) Oxidizer massflow cylindrical port (grain 1)

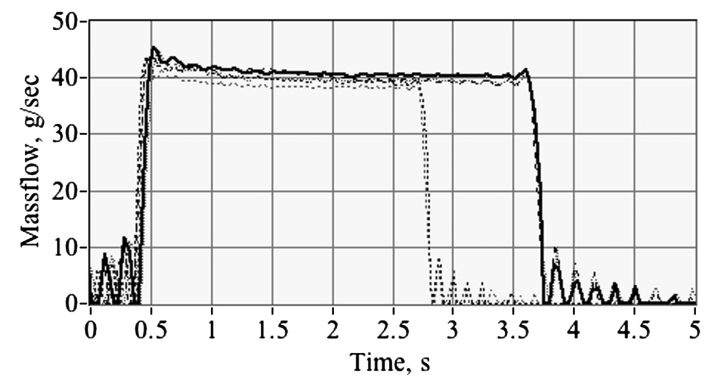

b) Oxidizer massflow helix ratio $=\mathbf{1 0 . 3 8}($ grain 2$)$

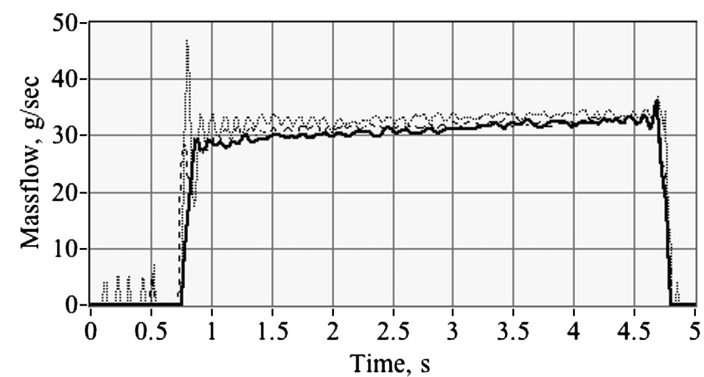

e) Fuel massflow cylindrical port (grain 1)

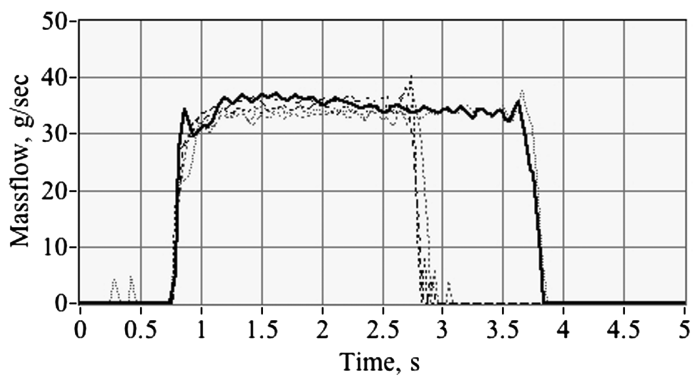

f) Fuel massflow helix ratio $=10.38$ (grain 2) files obtained from this test series. Figures $\underline{4 a}-4 d$ plot the thrust time history profiles obtained for each of the test geometries listed by Table 1 . Figures $\underline{4 \mathrm{e}}-4 \mathrm{~h}$ plots the chamber pressure profiles. The time histories corresponding to the first burns of the test geometries are plotted as solid black lines. Time histories from the subsequent burns are plotted as lighter, dashed lines. The most distinguishing feature to note is that, although the cylindrical port thrust and chamber pressure levels are quite consistent and nearly flat from burn to burn, the helical port runs show quite a bit of burn-to-burn variability, with the thrust and chamber pressure levels dropping off with time for the first burn (solid line) and tapering to fairly flat for the successive burns.

Figure 5 presents corresponding mass flow time history plots, with Figures $5 \mathrm{a}-5 \mathrm{~d}$ plotting oxidizer mass flow rate and Figs. $5 \mathrm{e}-5 \mathrm{~g}$ plotting the fuel mass flow rate. The fuel mass flow rate was calculated as the difference between the Venturi-measured oxidizer mass flow and the nozzle exit mass flow. The nozzle exit mass flow was calculated using the measured chamber pressure, flame temperature, nozzle exit area, and the one-dimensional choking mass flow equation [43]:

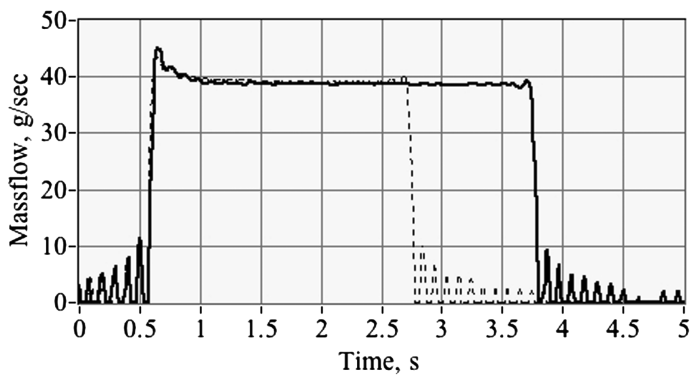

c) Oxidizer massflow helix ratio $=5.57$ (grain 3)

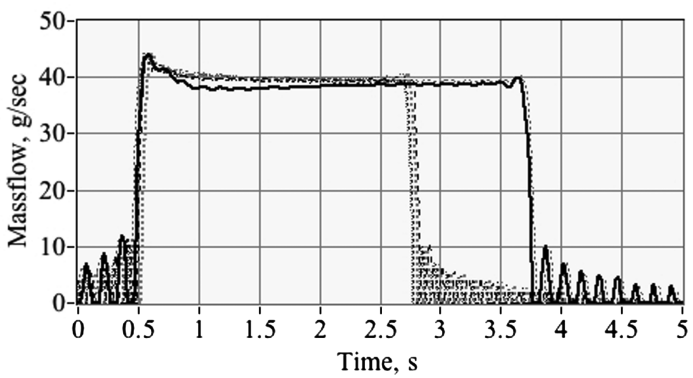

d) Oxidizer massflow helix ratio $=0.59($ grain 4$)$

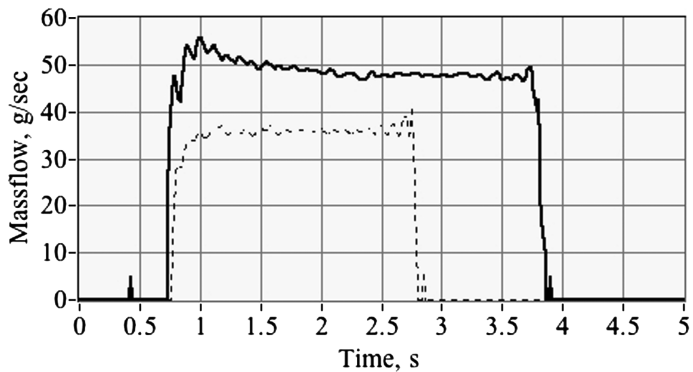

g) Fuel massflow helix ratio $=5.57($ grain 3)

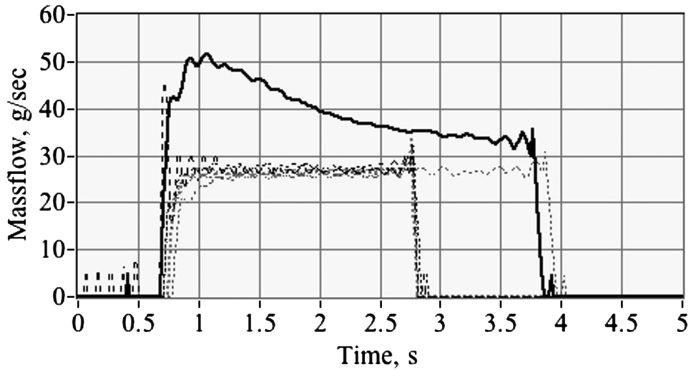

h) Fuel massflow helix ratio $=0.59$ (grain 4)

Fig. 5 GOX-ABS regression tests, oxidizer, and fuel mass flow rate time histories. 


$$
\dot{m}_{\text {propellant }}=A^{*} \cdot \frac{P_{0}}{\sqrt{T_{0}}} \cdot \sqrt{\frac{\gamma}{R_{g}} \cdot\left(\frac{2}{\gamma+1}\right)^{\gamma+1 / \gamma-1}}
$$

In Eq. (15), $T_{0}$ is the combustion flame temperature, and $R_{g}$ and $\gamma$ are the gas constant and ratio of specific heats of the combustion products. As previously described, the thermodynamic and masstransport properties of the combustion products were calculated by interpolating tables developed from the NASA chemical equilibrium program Chemical Equilibrium with Applications (CEA) [44]. The CEA tables were interpolated using the measured chamber pressure and an assumed $O / F$ ratio as inputs. To ensure that biases due to the exhaust product gas properties were minimized, the $O / F$ ratio entered into the CEA tables was adjusted to produce a fuel mass flow whose integrated value over the burn time exactly equals the consumed fuel mass as measured after each burn test. Notice that, although the oxidizer mass flow remains very consistent from burn to burn as a result of the choked injector, the consumed fuel mass flow varies significantly, indicating significant changes in the burn $O / F$ ratio and fuel regression rate.

Figure 6 plots the $O / F$ ratio as a function of burn time for the tested geometries listed in Table 1 . The resulting profiles are extremely interesting. First, the cylindrical port shows a slight drop in the $O / F$ ratio as a function of burn time with values that vary from an initial $O / F$ of 1.5 to less than 1.2 by the completion of the final burn. Next, the "moderate" helical fuel import with an initial helix ratio of 10.38 shows almost no variation of $O / F$ as a function of burn time but does show a mean $O / F$ ratio that is measurably lower than the cylindrical port grain, approximately 1.18 . Finally, the very "aggressive" helical ports show initial $O / F$ ratios that are significantly lower for the initial burn but that lowered $O / F$ rapidly grows as a function of burn time. The very short-pitch-length fuel grain (4) shows the most rapid growth in $O / F$ ratio. In fact, after the first $3 \mathrm{~s}$ burn, grain 4 shows a mostly flat $O / F$ variation as a function of burn time.

\section{Estimating the Fuel Regression Rates and Mean Port Mass Flux}

The longitudinal fuel regression rate was calculated from the fuel mass flow starting with the regression rate definition:

$$
\overline{\dot{r}}=\frac{\dot{m}_{\text {fuel }}}{\rho_{\text {fuel }} \cdot 2 \pi \cdot \bar{r} \cdot L}
$$

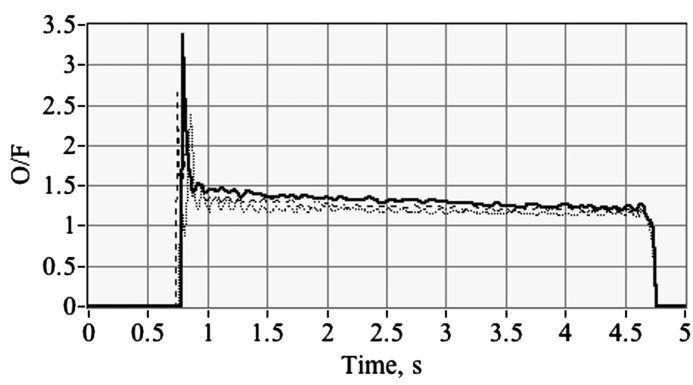

a) $\mathrm{O} / \mathrm{F}$ for cylindrical port

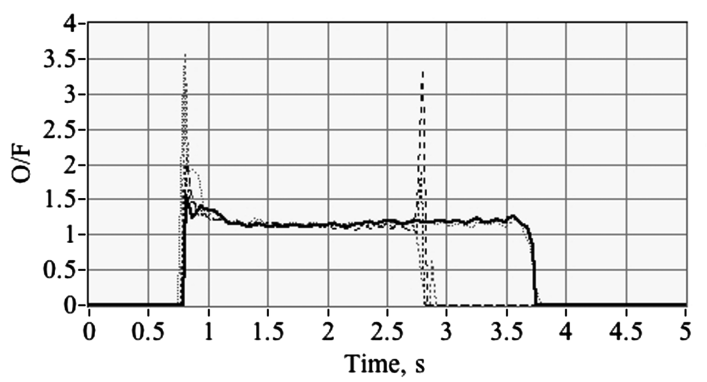

b) $\mathrm{O} / \mathrm{F}$ for helix ratio $=10.38$ (grain 2)
Rearranging Eq. (16) and integrating from the initial condition to the current time solves for the mean instantaneous fuel port diameter:

$$
\bar{r}(t)=\sqrt{r_{0}^{2}+\int_{0}^{t} \frac{\dot{m}_{\text {fuel }} \cdot \mathrm{d} t}{\rho_{\text {fuel }} \cdot \pi \cdot L}}
$$

The longitudinal mean of the total mass flux is estimated as

$$
\bar{G}_{\text {total }}=\frac{\dot{m}_{\text {ox }}+5 / 9 \cdot \dot{m}_{\text {fuel }}}{\pi \cdot r_{0}^{2}+\int_{0}^{t} \dot{m}_{\text {fuel }} \cdot \mathrm{d} t / \rho_{\text {fuel }} \cdot \pi \cdot L}
$$

In Eq. (18), the scaling factor of 5/9 on the fuel mass flow results when the classical Marxman regression rate equation $[25,26]$ is integrated along the length of the fuel port to account for contributions of the ablated fuel mass flow to the overall fuel port mass flux [45].

\section{Comparing the Fuel Regression Rates for the Helical and Straight-Port Geometries}

Using the data of Figs. $\underline{5}$ and $\underline{6}$, Eqs. (16-18) are used to calculate the instantaneous mean regression rate profile for each of the test burns and the instantaneous mean mass flux profiles. Figure 7 shows the result where the plotted symbols represent the regression rate data calculated from the test burns, and the plotted lines represent the predicted regression rate calculated using the classical Marxman model as modified by Eilers and Whitmore [46] for the straight-bore port (grain 1) and scaled-values of this curve for each of the helical geometries (grains 2, 3, and 4) calculated using the Mishra-Gupta model of Eq. (14). The Mishra-Gupta extrapolations assume constant values for $\bar{d}, P$, and $\eta_{h}$ but allow the instantaneous port diameter $D$ to grow as the fuel port burns.

\section{Interpretation of Fuel Regression Rate Observations}

Even though the data presented by Fig. 7 were collected from multiple burns of each fuel grain, the empirical data tend to group into 4 distinct curves, one for each of the initial test geometries. Initially, the helical port data exhibit a large amplification factor, but as the port burns, the port cross section becomes more and more cylindrical, and the helical effects on skin friction diminish with time. Clearly, the helix pitch ratio has very significant effect on the regression rate.

The Mishra-Gupta extrapolations tend to underpredict the regression rate at high flux levels and overpredict the regression rate at

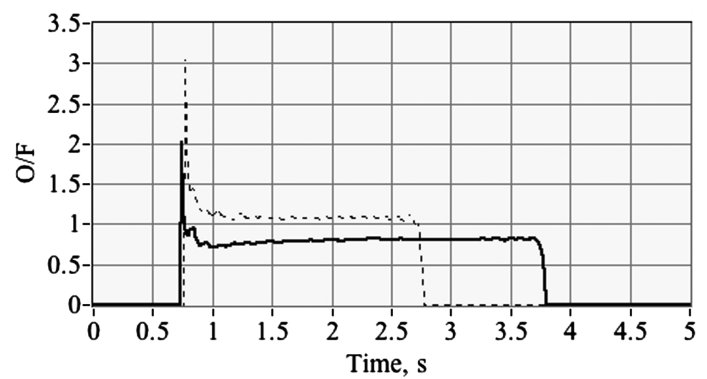

c) $\mathrm{O} / \mathrm{F}$ for helix ratio $=\mathbf{5 . 5 7}($ grain 3)

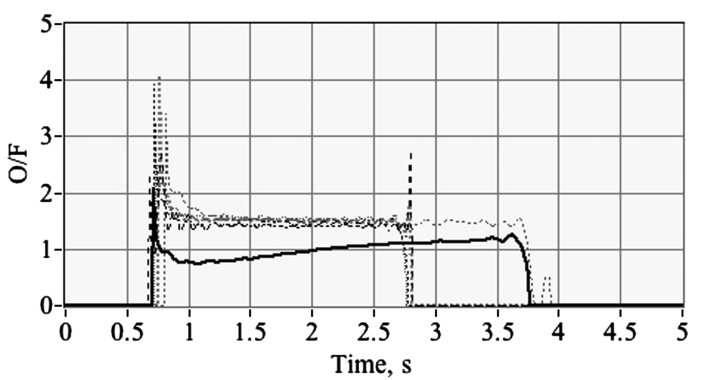

d) $\mathrm{O} / \mathrm{F}$ for helix ratio $=0.59($ grain 4$)$

Fig. 6 GOX-ABS regression tests, variation in $O / F$ ratio with burn time. 


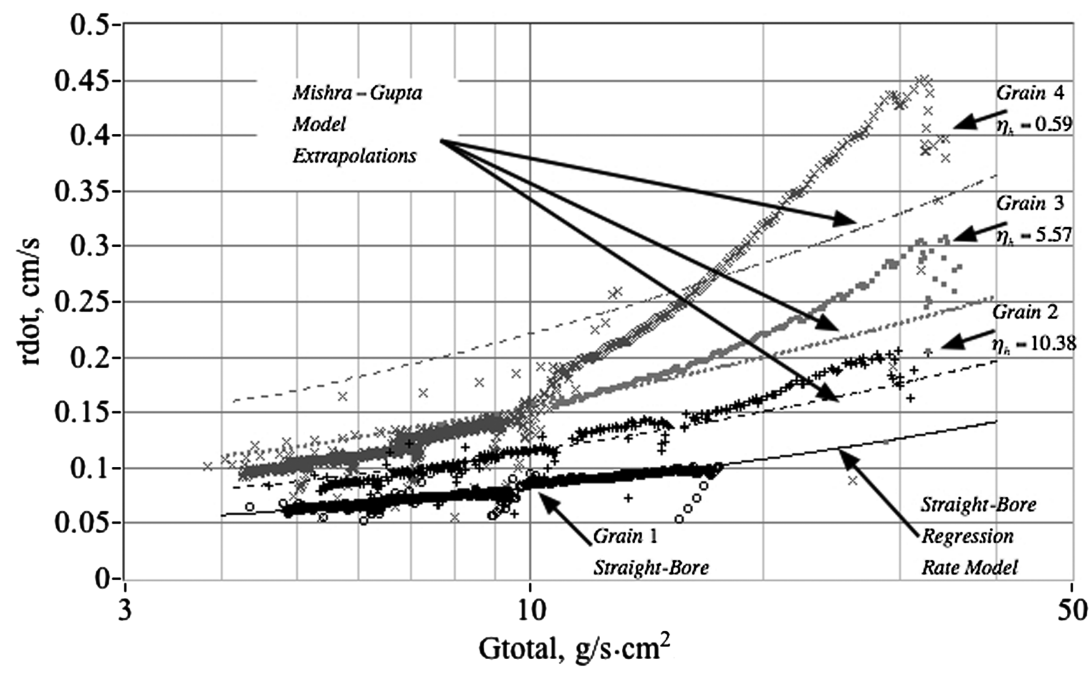

Fig. 7 GOX-ABS regression rate comparison for straight-bore and helical grains.

lower flux levels. Recall that the Mishra-Gupta model does not account for radial outflow of the ablated fuel mass flow, and the extrapolated curves of Fig. 7 model the direct effects of the helix on skin friction only. Thus, the observed differences between the observed and predicted regression rate trends present clear evidence that blowing effect suppression is a significant factor in the regression rate amplification due to the helical fuel port, with a magnitude that is at least equivalent to the increase in the skin friction coefficient.

In these graphs, the high mass flux rates occur early in the burn sequences where the fuel port is still quite helical, and the resulting centrifugal flow effects push the flame zone closer to the wall. As the port burns and the mass flux drops, the helical structures also regress both radially and longitudinally, gradually becoming almost cylindrical in shape. The resulting drop in centrifugal flow allows radial blowing to push the flame zone away from the wall, and the regression rate drops significantly. The faster the initial regression rate is, the more rapidly the port becomes cylindrical; thus, the short pitch length fuel grain where the helical loops are spaced very close together longitudinally shows the most significant drop in regression rate.

It is also informative to plot the regression rate against the rotational Reynolds number because that parameter provides both a measure of the centrifugal and skin friction effects of the helical flowfield. For this analysis, the rotational Reynolds number is calculated using the mean total port mass flux, and it can be shown that

$$
R_{e_{\omega}}=\frac{\rho \cdot\left(\omega \cdot R_{c}\right) \cdot S}{\bar{\mu}}=2 \pi \cdot\left(\frac{R_{c}}{P}\right) \cdot\left(\frac{L}{\bar{\mu}}\right) \cdot \bar{G}_{\text {total }}
$$

In Eq. (8), $\omega$ is the rotational rate of the flow in the chamber, $\bar{\mu}$ is the mean viscosity of the combustion by products, and $S$ is the length of the helix centerline:

$$
S=2 \pi \cdot N \cdot \sqrt{\left(\frac{\mathrm{d}}{2}\right)^{2}+\left(\frac{P}{2 \pi}\right)}
$$

Figure 8 presents the results of this calculation. Curve fits of the data are also plotted showing the extrapolation down to essentially zero rotational Reynolds number. All three curves for the helical fuel grains asymptotically approach the baseline regression rate value extrapolated from the data of Fig. 7 at low oxidizer mass flux. This result strongly supports the hypothesis that the rotational flow suppression of the fuel blowing effect is a primary driver for the observed regression rate amplification.

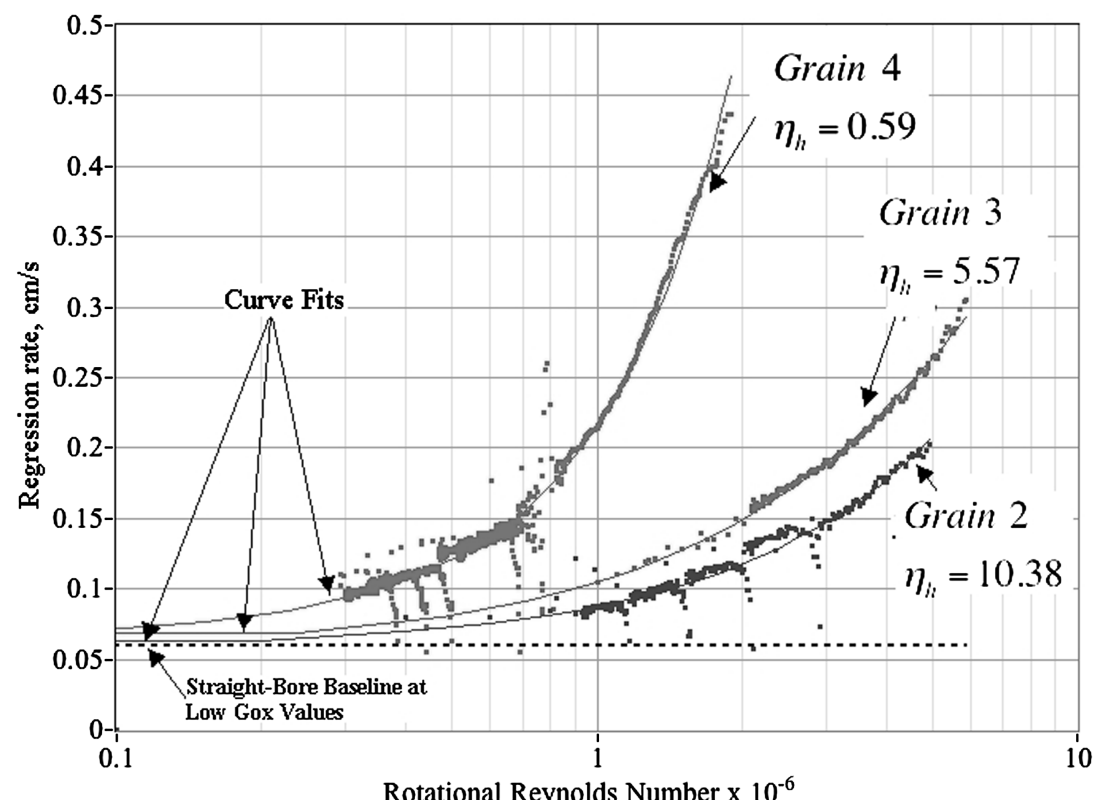

Fig. 8 Effect of rotational Reynolds number on fuel regression rate. 


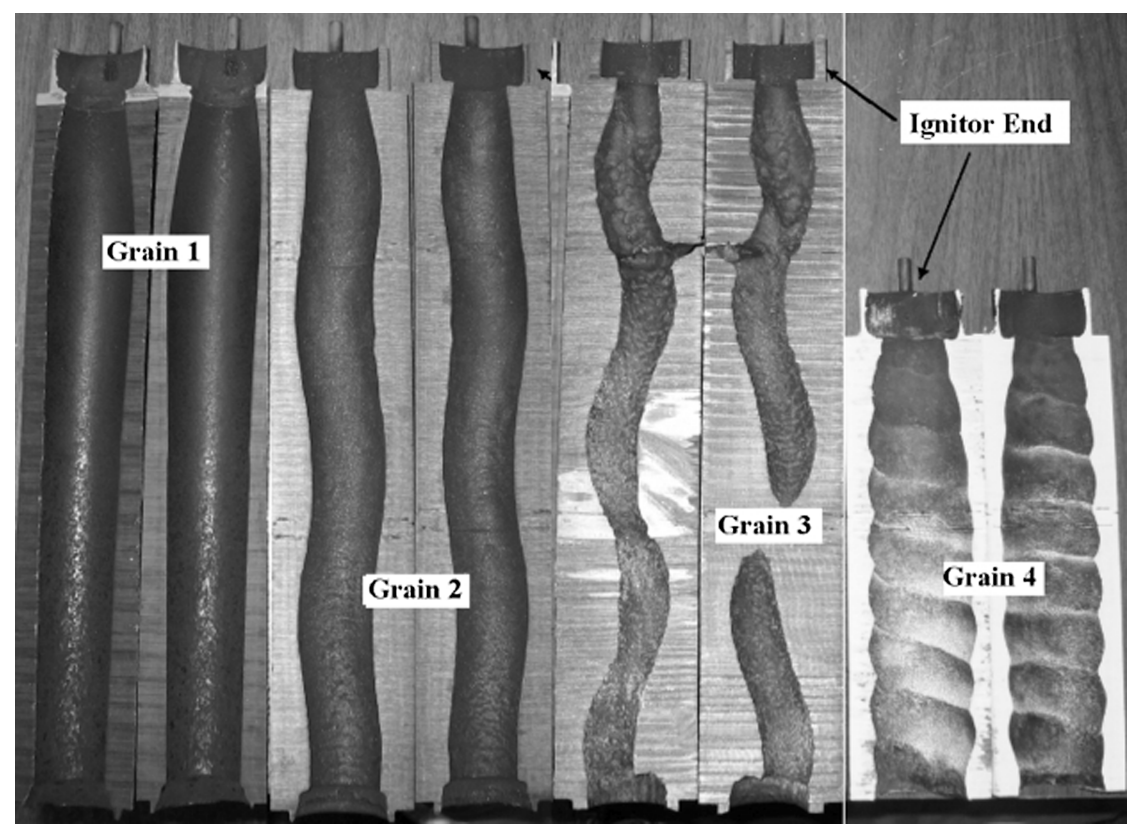

Fig. 9 Posttest fuel grain cross sections.

\section{Posttest Fuel Grain Observations}

Following the testing campaign, each of the fuel grains were cut longitudinally along the port centerline to inspect the residual fuel port structures. Figure 9 shows the resulting cross sections. These images support the earlier assertion that the thrust and chamber pressure differences between the successive helical-port time history plots are due to the changing fuel regression rate as the port helix opens up and the helical flow effects diminish. In fact, the short pitch length (grain 4) cross section has become nearly cylindrical. Grain 3 retained the most posttest helical structure and thus exhibited the least drop off in regression rate. As the fuel grain burns and the helix ratio becomes small, the burn pattern becomes progressively more like a cylindrical fuel grain. These observations agree with the Eqs. (11, and 12) helical port model discussion presented in Sec. II.B. As described earlier, conveniently, this effect reduces the potential for excessive unburned fuel "slivers" at the end of the burn lifetime of the fuel grain.

\section{Conclusions}

Hybrid rockets produce fuel regression rates are typically $25 \%$ lower than solid propellant motors in the same thrust class. These lowered regression rates produce unacceptably high oxidizer-to-fuel $(O / F)$ ratios and can result in motor instability, nozzle erosion, and reduced motor duty cycles. Several methods including multiple fuel ports, liquefying fuel materials, and grain metallization have been previously investigated to increase regression rates, but each method has significant developmental issues.

This paper presents an alternative approach that uses helical fuel port geometries to modify the internal fuel port flow properties and increase the fuel regression rate. These helical port structures are enabled using additive manufacturing. The helical fuel port also increases the volumetric efficiency of the fuel grain by lengthening the internal flow path for a given fuel port length. An order of magnitude analysis of a simple helical flow model, based upon the skin friction correlation developed by Mishra and Gupta for helical pipe flows, predicts a significant potential for the helical port to significantly increase the overall fuel regression rate.

To verify these order of magnitude predictions, a series of static firings of a midsized lab-scale hybrid motor with multiple helical fuel port geometries were performed. In this series of tests, the helical ports were fabricated with an initial ratio of the radius of curvature to port radius varying from greater than 10 (a "mild" helix) to approximately $1 / 2$ (an aggressive helix). The resulting regression rates were compared to those obtained for the same motor with a cylindrical port of the same initial port radius. For this series of tests, gaseous oxygen is used as the oxidizer to allow a choked injector and thus ensure that the desired oxidizer mass flow rate is decoupled from the fuel regression rate. The additively manufactured fuel grains were constructed from acrlyonitrile butadiene styrene (ABS) using conventional 3-D printer stock material.

The presented results demonstrate that the centrifugal flow patterns introduced by the helical fuel port increases fuel regression rates significantly. Mean regression rate amplification factors as a function of oxidizer mass flux of greater than 4 are observed for the very aggressive helical port structures with short pitch distances. Even the very modest helical ports were shown to achieve mean amplification factors of greater than 2 . The presented analysis also demonstrates that the rotational flow velocity within the helical fuel port is a primary driver for the regression rate amplification, at least equivalent in magnitude to the increase in skin friction caused by the helical fuel port. Because the rotational velocity of the flowfield diminishes with time as the fuel port opens up and becomes more and more helical, the regression rate amplification factor also drops with time.

Because ABS can be manufactured in an almost infinite variety of shapes using additive processes, there exists the potential to "draw" high regression rate ABS fuel grains that mathematically optimize desired combustion properties. One example is the design of a high regression rate port structure that minimizes the oxidizer-to-fuel ratio shift and maintains an optimal point on the characteristic velocity curve for a wide range of oxidizer mass flux levels. Based on the presented results, both the increase in local skin friction coefficient and the radial blowing suppression must be considered when developing an "optimal" port design. A current limitation of the helical fuel regression model based on the Mishra-Gupta correlation is the inability to account for this wall-blowing suppression. Clearly, the fidelity of the current helical regression rate model needs to be extended to account for both of these effects to allow detailed optimization studies. Fortunately, because the grains are built additively, if one can draw it, then one can build it, and this challenge appears to be quite tractable.

A suggested follow-on research activity is to reproduce the presented test matrix using a traditional hybrid propellant such as hydroxyl-terminated polybutadiene. Because very modest helices have been shown to produce significant effects, it should be possible using an appropriate mold-release agent to cast the fuel material around a helical mandrel and then remove that mandrel once the material cures. Such a method, when proven to be effective, would provide a simple and effective "drop-in" method to improve the performance of many legacy hybrid systems. The demonstrated 
regression rate amplification effects should also be demonstrated using an alternate two-phase oxidizer like nitrous oxide, where the injector will be only partially choked. Thus, the injector mass flow may couple with the observed fuel regression rate and may significantly affect the observed results.

\section{References}

[1] "Hazard Analysis of Commercial Space Transportation; Vol. 1,: Operations, Vol. 2: Hazards, Vol. 3: Risk Analysis," U.S. Dept. of Transportation, Rept. PB93-199040, May 1988.

[2] Cheng, C. G., Farmer, R. C., Jones, H. S., and McFarlane, J. S., "Numerical Simulation of the Internal Ballistics of a Hybrid Rocket," 30th AIAA/ASME/SAE/ASEE Joint Propulsion Conference \& Exhibit, AIAA Paper 1994-0554, July 1994.

[3] Karabeyoglu, M. A., Stevens, J., and Cantwell, B. J., "Investigation of Feed System Coupled Low Frequency Combustion Instabilities in Hybrid Rockets," 43rd AIAA/ASME/SAE/ASEE Joint Propulsion Conference \& Exhibit, AIAA 2007-5366, July 2007.

[4] Sutton, G. P., and Biblarz, O., Rocket Propulsion Elements, 7th ed., Wiley, New York, 2001, pp. 579-606.

[5] Karabeyoglu, M. A., "Hybrid Rocket Propulsion for Future Space Launch," Stanford Univ. Aero/Astro 50th Year Anniversary Presentation, May 2008, http://aa.stanford.edu/events/50thAnniversary/ media/Karabeyoglu.pdf [retrieved 23 Dec. 2013].

[6] Fiedler, R. A., Breitenfeld, M. S., Jiao, X., Haselbacher, A., Geubelle, P., Guoy, D., and Brandyberry, M., "Simulations of Slumping Propellant and Flexing Inhibitors in Solid Rocket Motors," 38th AIAA/ASME/SAE/ ASEE Joint Propulsion Conference \& Exhibit, AIAA Paper 2002-4341, 2002.

[7] Pastrone, D., "Approaches to Low Fuel Regression Rate in Hybrid Rocket Engines," International Journal of Aerospace Engineering, Vol. 2012, 2012, p. 12. doi:10.1155/2012/649753

[8] Ebeling, R. W., Jr., "Non-Sustaining Hybrid Propellant Grain," U.S. Patent No. 3698191, Oct. 1972.

[9] Sutton, G. P., and Biblarz, O., Rocket Propulsion Elements, Wiley, New York, 2001, pp. 520-537.

[10] Knuth, W. H., Chiaverini, M. J., Sauer, J. A., and Gramer, D. J., "SolidFuel Regression Rate Behavior of Vortex Hybrid Rocket Engines," Journal of Propulsion and Power, Vol. 18, No. 3, 2002, pp. 600-609. doi:10.2514/2.5974

[11] McDonald, M., and Badescu, V., The International Handbook of Space Technology, Springer-Verlag, Berlin, 2014, p. 300.

[12] Boardman, T. A., Hawkins, D. K., Wassom, S. R., and Claflin, S. E., "Nonacoustic Feed System Coupled Combustion Instability in Hybrid Rocket Motors," Presentation at the Hybrid Rocket Technical Committee Combustion Stability Workshop, 31st AIAA/ASME/SAE/ ASEE Joint Propulsion Conference, July 1994, http://ntrs.nasa.gov/ archive/nasa/casi.ntrs.nasa.gov/19990101870.pdf [retrieved $14 \mathrm{Feb}$. 2015].

[13] Park, K. S., and Lee, C., "Low Frequency Instability in LaboratoryScale Hybrid Rocket Motors," Aerospace Science and Technology, Vol. 42, No. 3, April-May 2015, pp. 148-157. doi:10.1016/j.ast.2015.01.013

[14] Karabeyoglu, M. A., and Arkun, U., "Evaluation of Fuel Additives for Hybrid Rockets and SFRJ Systems," 50th AIAA/ASME/SAE/ASEE Joint Propulsion Conference and Exhibit, AIAA Paper 2014-3647, July 2014

[15] Lee, T. S., and Potapkin, A., "The Performance of a Hybrid Rocket with Swirling GOX Injection," Aerospace Science and Technology Research Center, Rept. ADA-409123, Aug. 2002. http://www.dtic.mil/cgi-bin/ GetTRDoc?AD=ADA409123 [retrieved 14 Feb. 2014].

[16] Knuth, W. H., Chiaverini, M. J., Sauer, J. A., and Gramer, D. J., "SolidFuel Regression Rate Behavior of Vortex Hybrid Rocket Engines," Journal of Propulsion and Power, Vol. 18, No. 3, 2002, pp. 600-609. doi: $10.2514 / 2.5974$

[17] Karabeyoglu, M. A., Altman, D., and Cantwell, B. J., "Combustion of Liquefying Hybrid Propellants: Part 1, General Theory," Journal of Propulsion and Power, Vol. 18, No. 3, March 2002, pp. 610-620. doi: $10.2514 / 2.5975$

[18] Nakagawa, I., Hikone, S., and Suzuki, T., "A Study on the Regression Rate of Paraffin-based Hybrid Rocket Fuels," 45th AIAA/ASME/SAE/ ASEE Joint Propulsion Conference \& Exhibit, AIAA Paper 2009-4935, July-Aug. 2009.

[19] Karabeyoglu, M. A., Zilliac, G., Cantwell, B. J., Dezilwa, S., and Castellucci, P., "Scale-Up Tests of High Regression Rate Paraffin-Based Hybrid Rocket Fuels," Journal of Propulsion and Power, Vol. 20, No. 5 ,
Nov.-Dec. 2004, pp. 1037-1045.

doi: $10.2514 / 1.3340$

[20] Galfetti, L., Merotto, L., Biocchi, F., Maggi, F., and De Luca, L. T., "Ballistic and Rheological Characterization of Paraffin-Based Fuels for Hybrid Rocket Propulsion," 47th AIAA/ASME /SAE/ASEE Joint Propulsion Conference \& Exhibit, AIAA Paper 2011-5680, JulyAug. 2011.

[21] "Weapons Systems and Platforms (WP) Focus Area, Environmentally Friendly Sustainable Binder System for Energetic Materials," Strategic Environmental Research and Development Program (SERDP), Exploratory Development (SEED) Rept. BAA-13-0002, 2014.

[22] Whitmore, S. A., Peterson, Z. W., and Eilers, S. D., "Comparing Hydroxyl Terminated Polybutadiene and Acrylonitrile Butadiene Styrene as Hybrid Rocket Fuels," Journal of Propulsion and Power, Vol. 29, No. 3, May-June 2013, pp. 582-592. doi:10.2514/1.B34382

[23] Blom, H., Yeh, R., Wojnarowski, R., and Ling, M., "Detection of Degradation of ABS Materials via DSC," Journal of Thermal Analysis and Calorimetry, Vol. 83, No. 1, Jan. 2006, pp. 113-115. doi:10.1007/s10973-005-6977-0

[24] Fuller, J. K., Ehrlich, D. A., Lu, P. C., Jansen, R. P., and Hoffman, J. D., "Advantages of Rapid Prototyping for Hybrid Rocket Motor Fuel Grain Fabrication," 47th AIAA/ASME/SAE/ASEE Joint Propulsion Conference \& Exhibit, AIAA Paper 2011-5909, July-Aug. 2011.

[25] Marxman, G., and Gilbert, M., "Turbulent Boundary Layer Combustion in the Hybrid Rocket," Symposium (International) on Combustion, Vol. 9, No. 1, 1963, pp. 371-383. doi:10.1016/S0082-0784(63)80046-6

[26] Marxman, G. A., Wooldridge, C. E., and Muzzy, R. J., "Fundamentals of Hybrid Boundary Combustion," Progress in Astronautics and Aeronautics, Vol. 15, No. 1, 1964, p. 485.

[27] Geankoplis, C. J., Transport Processes and Separation Process Principles, 4th ed., 2003, p. 475.

[28] White, F. M., Viscous Fluid Flow, McGraw-Hill, New York, 1991, pp. $485-486$.

[29] McCabe, W. L., Smith, J. C., and Harriot, P., Unit Operations of Chemical Engineering, 7th ed., McGraw-Hill, 2004, pp. 369-370.

[30] Lees, L., "Convective Heat Transfer with Mass Addition and Chemical Reactions," Proceedings of Combustion and Propulsion: 3rd AGARD Colloquium, Pergamon, New York, 1958, p. 451.

[31] Sutton, G. P., and Biblarz, O., Rocket Propulsion Elements, Wiley, New York, 2001, pp. 731-737.

[32] White, C. M., "Friction Factor and Its Relation to Heat Transfer, Transactions of the Institution of Chemical Engineers," Transactions of the Institution of Chemical Engineers, Vol. 18, No. 1, 1932, pp. 66-86.

[33] Ito, H., "Friction Factor for Turbulent Flow in Curved Tube," Journal of Basic Engineering, Vol. 81, No. 1, 1959, pp. 123-134.

[34] Gnielinski, G. V., "Heat Transfer and Pressure Drop in Helically Coiled Tubes," Proceedings of 8th International Heat Transfer Conference, San Francisco, Vol. 6, No. 1, 1986, pp. 2847-2854

[35] Mishra, P., and Gupta, S. N., "Momentum Transfer in Curved Pipes. 1. Newtonian Fluids," Journal of Industrial Engineering and Chemical Process Development, Vol. 18, No. 1, 1979, pp. 130-137. doi:10.1021/i260069a017

[36] Bath, A., "Performance Characterization of Complex Fuel Port Geometries for Hybrid Rocket Fuel Grains," M.S. Thesis, College of Engineering and Applied Sciences, Utah State Univ., Logan, UT, Dec. 2012.

[37] Arnold, D., Boyer, J. E., Kuo, K. K., DeSain, J. D., Curtiss, T. J., and Fuler, J. K., "Test of Hybrid Rocket Fuel Grains with Swirl Patterns Fabricated Using Rapid Prototyping Technology," 49th AIAA/ASME/ SAE/ASEE Joint Propulsion Conference, AIAA Paper 2013-4141, July 2013.

[38] Whitmore, S. A., Inkley, N. R., and Merkley, D. P., "Development of a Power Efficient, Restart-Capable Arc Ignitor for Hybrid Rockets," 50th AIAA/ASME/SAE/ASEE Joint Propulsion Conference and Exhibit, AIAA Paper 2014-3949, July 2014.

[39] "Pro75, A Better Way to Fly," http://www.pro38.com/products/pro75/ pro75.php [retrieved 25 Dec. 2013].

[40] Whitmore, S. A., Inkley, N. R., and Merkley, D. P., "Development of a Power Efficient, Restart-Capable Arc Ignitor for Hybrid Rockets," 50th AIAA/ASME/SAE/ASEE Joint Propulsion Conference and Exhibit, AIAA Paper 2014-3949, July 2014.

[41] "Dimension 1200es, Durability Meets Affordability," http://www .stratasys.com/3d-printers/design-series/performance/dimension1200es [retrieved 25 Dec. 2013].

[42] "ABSplusTM-P430 Production-Grade Thermoplastic for Design Series 3D Printers," http://www.stratasys.com/ /media/Main/Secure/ Material-Specs-MS/Fortus-Material-Specs/Fortus-MS-ABSplus-01-13web.pdf [retrieved 12 Feb. 2014]. 
[43] Sutton, G. P., and Biblarz, O., Rocket Propulsion Elements, 7th ed., Wiley, New York, 2001, Chaps. 4-5.

[44] Gordon, S., and McBride, B. J., "Computer Program for Calculation of Complex Chemical Equilibrium Compositions and Applications," NASA, RP-1311, 1994

[45] Whitmore, S. A., "Introduction to Hybrid Rockets," Classroom Notes, pp. 61-70, http://www.neng.usu.edu/classes/mae/6530/propII/New_ Course/Section3/section3.1.pdf [retrieved 25 Oct. 2014].
[46] Eilers, S. D., and Whitmore, S. A., "Correlation of Hybrid Rocket Propellant Regression Measurements with Enthalpy-Balance Model Predictions," Journal of Spacecraft and Rockets, Vol. 45, No. 4, Sept.Aug. 2008, pp. 1010-1020.

doi: $\underline{10.2514 / 1.33804}$ 


\section{Queries}

1. AU: Please check that the authors' affiliations and footnotes are correct.

2. AU: Please check that the copyright (@) information is correct.

3. AU: Acronyms are not allowed in the Abstract.

4. AU: Definitions of acronyms are not allowed in the Nomenclature.

5. AU: The Nomenclature was reordered according to AIAA style: alphabetically (capital letters before lowercase), English then Greek.

6. AU: Arrows are not allowed above math terms. These have been set in boldface italic. Please confirm that your intended meaning is retained.

7. AU: The first section must be called "Introduction".

8. AU: Please note that subsections are not allowed in the Introduction and have been removed. If you prefer, you may move these subsections to a new Sec. II, with subsequent sections renumbered appropriately.

9. AU: Please spell out "AMROC".

10. AU: Acronyms that are defined and used only once (e.g., PBAN, GAP) are removed.

11. AU: The name given in the text for Ref. [31] (Boardman) does not match the names given in the list of references (Sutton and Biblarz). Please change one or both so that they match.

12. AU: Acronyms are not allowed in section headings.

13. AU: The final section must be called "Conclusions".

14. AU: If Ref. [5] is a published proceedings, please provide the name and location of the publisher (NOT of the conference host) and the page numbers used. If it is a conference paper, please provide the paper number and the organizer's name. If it is a CD$\mathrm{ROM}$, please provide the name and location of the CD-ROM producer.

15. AU: Please provide issue numbre and/or month of publication as well as complete page range for Ref. [7].

16. AU: If Refs. [12, 20,34] are published proceedings, please provide the names and locations of the publishers (NOT of the conference hosts) and the page range. If they are conference papers, please provide the paper numbers and the organizers' names. If they are CD-ROMs, please provide the names and locations of the CD-ROM producers.

17. AU: Please provide the location of the institution for Ref. [15].

18. AU: Please provide the name and location of the institution for Ref. [21].

19. AU: Please provide complete page range for Ref. [26].

20. AU: Please provide the name and location of the publisher for Ref. [27]. 
21. AU: Please provide the location of the publisher for Ref. [29].

22. AU: Please provide complete page range for Ref. [30].

23. AU: To use a URL as a reference, please provide name and location of publisher as well as date of publication. If this information cannot be provided, or if the URL does not link to a publisher paper, report, etc., then please convert Refs. [39, 41, $42,45]$ to a footnote.

24. AU: The URL provided for Ref. [42] does not work. Please provide a valid URL and date of retrieval for this reference. Please also see the previous comment regarding URL references.

25. AU: Please provide the page range for Ref. [43]. 